\title{
A Novel Compact Dual-Band Slot Antenna
}

\section{Sahar Mardani \\ Ghahfarokhi}

Department of Communication, Bushehr Branch, Islamic Azad University Bushehr, Iran.

\author{
Alireza Mallahzadeh \\ Department of \\ Communication,khalij fars \\ University,Bushehr,iran
}

\author{
Sara Jafarpoor \\ Department of Communication, \\ Bushehr Branch, \\ Islamic Azad University \\ Bushehr, Iran.
}

\begin{abstract}
This paper introduces a compact dual-band slot antenna using spiral inductive loaded resonators. The radiating elements of the proposed antenna are composed of two balanced short circuited slot lines. To design a dual-band antenna, the microstrip feed line is somehow implemented to provide three short circuit points between the antenna and the feed lines. The antenna size is very compact, and can be integrated easily with other RF front-end circuits for dual-band applications. It is demonstrated that the proposed antenna can provide dual-band behavior with satisfactory radiation characteristics.
\end{abstract}

\section{Keywords}

Dual band antenna, high Frequency antenna, spiral antenna, slot antenna, parasitic Couplings

\section{INTRODUCTION}

The spiral antenna geometry has been subject of investigations for several years by which the size of such elements can be significantly reduced. The main characteristics of the spiral antenna are its broad bandwidth of operation, wide half power beam width (HPBW), and circular polarization (CP) for the radiated field [1]-[3]. These characteristics are attractive for wireless systems such as wireless-LAN, GSM, CDMA, etc., [3][6]. One characteristic of the spiral antenna utilized in this paper is the fact that by adding the further inductive loaded to its arms and exciting

the slot in two different points, a dual-band antenna can be designed and fabricated.

Several different dual-band slot antennas have been reported in the past few years [4-8], while these dual-band antennas realize dual-band behavior, they generally occupy a relatively large area on their substrates.

As stated, spiral antenna topologies are good candidates to reduce the size of such elements. In this paper, the proposed antenna in [9] and [10] are considered and based on their topologies a novel compact dual-band

antenna is designed and proposed. The proposed antenna has the same size, but with dual-band behavior. Furthermore the proposed antenna is designed and simulated on a substrate with thickness of $500 \mathrm{~m}$, a dielectric constant of 3.5, and a loss tangent of 0.003 with a copper ground plane of $33.5 \times 23 \mathrm{c}$

\section{THE PROPOSED STRUCTURE}

To present the proposed topology of the dual-band slot antenna, the structure of the conventional single-band slot antennas are initially followed. As indicated in [10], the conventional singleelement antenna provides a narrow band one with a BW of 8 $\mathrm{MHz}$, whereas the double-element one has been developed to enhance the bandwidth providing a BW of $21.6 \mathrm{MHz}$.
As described in [10], the series inductors have been used to reduce the size of antenna, these series inductors have been put in the arms of the main slot where the amplitude of magnetic current is small. In this paper, by taking inspiration of this technique, a series inductor is somehow located to the main slot to create a self- parasitic coupling between it and the main slot. Since the magnetic current has its maximum value in the center of the slot, it is the best location to put the series inductor and creating self-parasitic coupling in the structure. Moreover, the impedance of a short circuited slot line can be expressed as follows:

$$
Z_{S}=j Z_{0 S} \tan \left(\beta_{g} 1\right)
$$

Where $\beta_{g}$ is the propagation constant, $Z_{0 S}$ is the characteristic impedance, and 1 is the length of the short circuited slot-line. It is well known that the characteristic impedance of a slot-line is inversely proportional to its width [11], thus, more inductance can be obtained for a fixed length of short circuited transmission line by using wider series slots.

In the light of these considerations, a series wide-width inductor is added to the center of the main slot and the topology indicated in Fig. 1 a is realized.

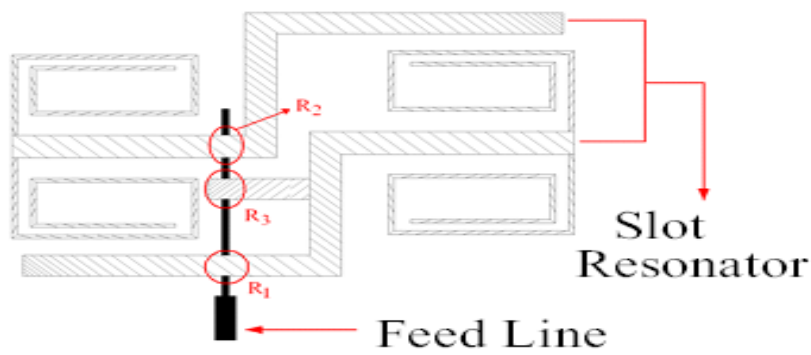

(a)

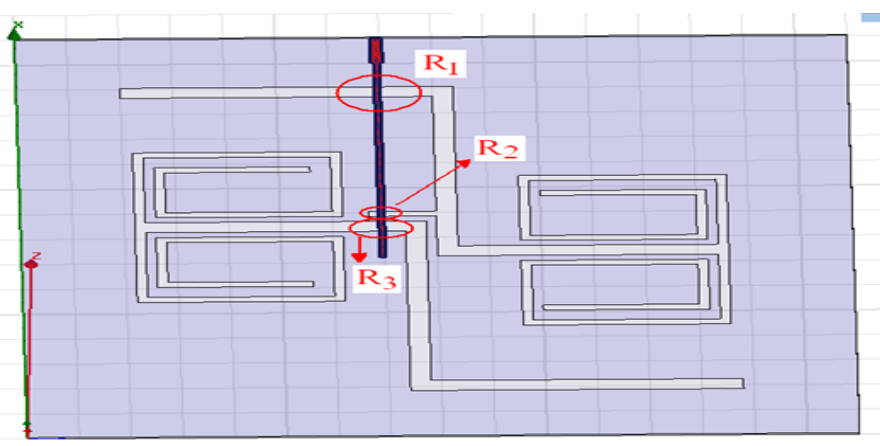

(b)

Fig 1: The diagram schematic of the (a) proposed singleelement slot antenna (b) dual-element slot antenna 
As shown in the proposed topology of the slot antenna, the series inductor is somehow located to have maximum area of coupling between it and the main slot.

Considering the useful technique presented in [10], to enhance the bandwidth and also reduce the overall size of the antenna, a double-element antenna can be designed by locating two singleelement ones closed to each other. Fig. 1 b shows a doubleelement slot antenna utilizing the proposed topology.

As demonstrated in [10], by arranging the two single-element antenna given in Fig. 1 b, the overall bandwidth of the structure can be increased, however, here is used from this characteristic of the spiral antenna to provide dual-band behavior. Considering the proposed dual-element slot antenna shown in Fig. $1 \mathrm{~b}$ and somehow increasing the feed point by making the microstrip feed line short-circuit with the slotline in two points, a dual-band antenna is realized. Fig. $1 \mathrm{~b}$ shows the final schematic diagram of the proposed dual-band slot antenna where there are two different short-circuit points between microstrip feed line and the double-element slot antenna.

Observing the topology of the proposed dual-band antenna, the characteristic impedances of the microstrip feed line are chosen based on the design considerations demonstrated in [10].

\section{SIMULATED RESULTS OF DUAL-BAND ANTENNA}

The proposed slot antenna with series inductors is simulated using HFSS simulator on a $500 \mathrm{~m}$ thick substrate. Fig. 2 a shows the simulated and measured return losses for the slot antenna with inductive loading.

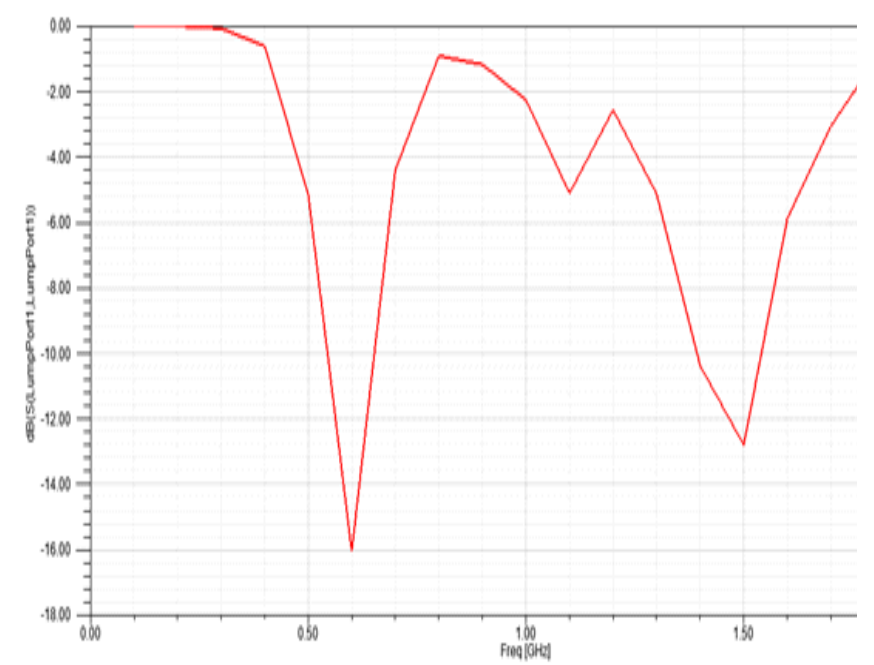

Fig 2: the simulated return loss of the proposed antenna

Comparing the obtained return loss for the proposed antenna and the conventional one, it is observed that the proposed one provides a dual-band one. As shown in Fig.2 , a return loss is achieved lower than $-10 \mathrm{~dB}$ in two different bands.

The proposed antenna provides dual-band with the resonant frequencies including the first one equal to $\mathrm{f}_{1}=786.5 \mathrm{MHz}$ and $\mathrm{f}_{2}$ $=813 \mathrm{MHz}$.

Fig. 3 shows the E- and H-plane co- and cross-polarized radiation patterns of the dual-band proposed loaded antenna at the first band. It is seen that the cross polarization level is negligible at broadside similar to the conventional one. Far field radiation patterns of proposed loaded slot antenna for the first resonant frequency are shown in Fig. $3 \mathrm{a}$ and $\mathrm{b}$ and those for the second resonant frequency are provided in Fig. $4 \mathrm{a}$ and $\mathrm{b}$.

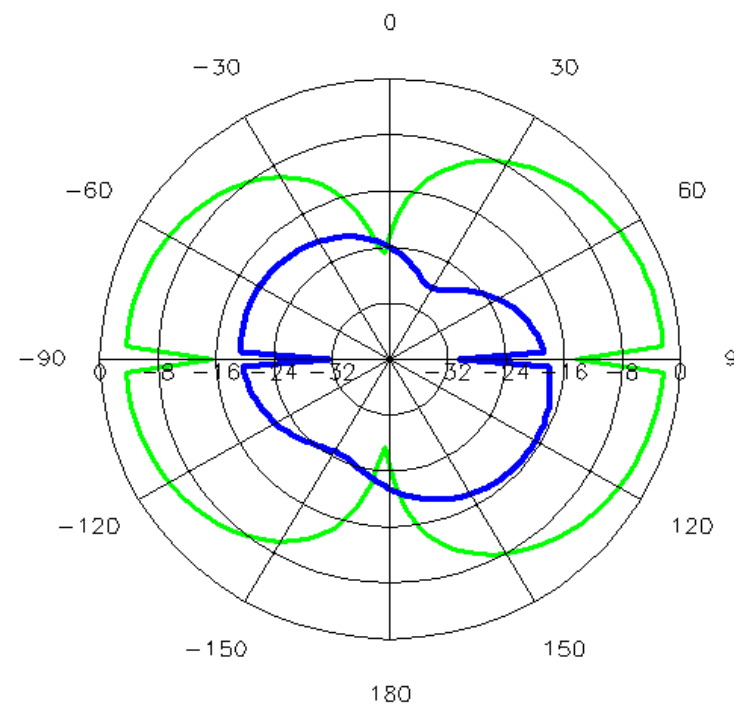

(a)

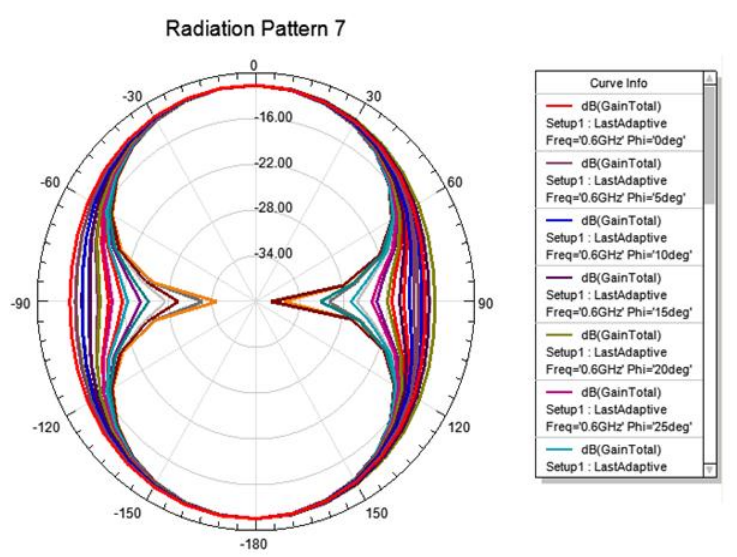

(b)

Fig 3: (a) and (b) far field radiation patterns of proposed loaded slot antenna at the first resonant frequency

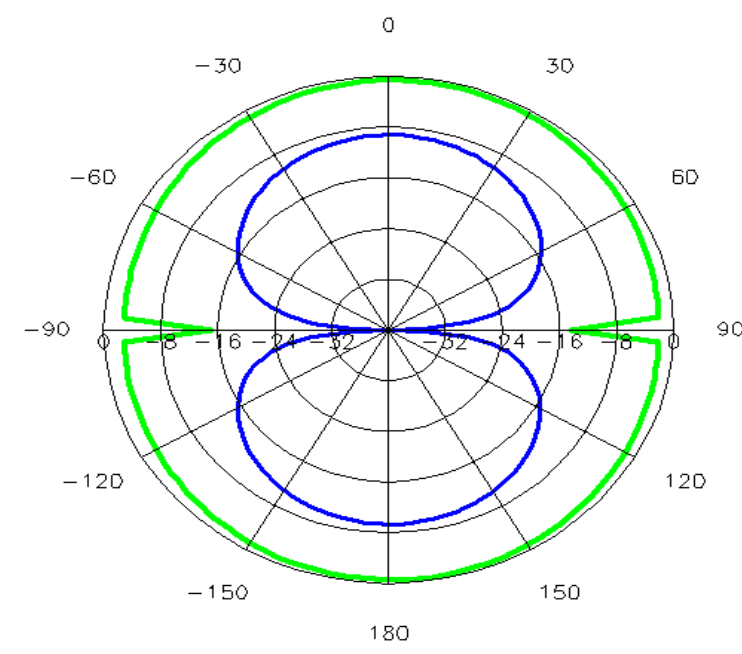

(a) 


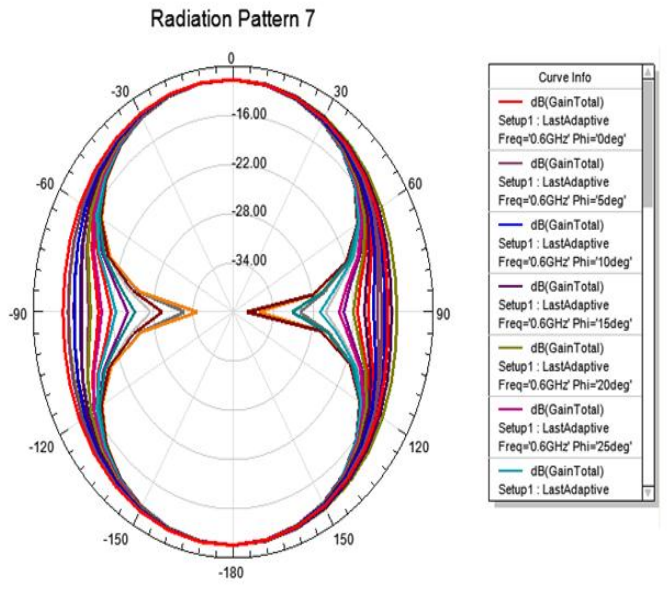

(b)

Fig.4 (a) and (b) far field radiation patterns of proposed loaded slot antenna at the second resonant frequency

\section{CONCLUSION}

A compact dual-band slot antenna has been designed and developed using spiral inductive loaded resonators in this paper. The radiating elements of the proposed antenna are reported to compose of two balanced short circuited slot lines. To design a dual-band antenna, the microstrip feed line has been implemented to provide three short circuit points between the antenna and the feed lines. The antenna size is very compact, and can be integrated easily with other RF front-end circuits for dualband applications. It has been shown that the proposed antenna can provide dual-band behavior with satisfactory radiation characteristics.

\section{REFERENCES}

[1] R. Bawer and J. J. Wolfe, "The spiral antenna," IRE Int. Convention Record, pt. T., pp. 84-95, 1960.
[2] J. A. Kaiser, "The archimedean two-wire spiral antenna," IRE Trans. Antennas Propag., vol. AP-8, pp. 312-323, May 1960.

[3] C. A. Balanis, Antenna Theory: Analysis and Design. New York: Wiley, 1997.

[4] Su, C. M., H. T. Chen, F. S. Chang, and K. L. Wong, "Dualband slot antenna for 2.4/5.2 GHz WLAN operation," Microwave and Optical Technology Letter, , Vol. 35, 306308, 2002.

[5] Wu, J. W., "2.4/5-GHz dual-band triangular slot antenna with compact operation," Microwave and Optical Technology Letter, Vol. 45, 81-84, 2005.

[6] Hsiao, H. M., J. W. Wu, and Y. D. Wang, "Novel dualbroadband rectangular-slot antenna for $2.4 / 5-\mathrm{GHz}$ wireless communication," Microwave and Optical Technology Letter, Vol. 46, 197-201, 2005.

[7] Chen, J. S., "Dual-frequency annular-ring slot antennas fed by CPW feed and microstrip line feed," IEEE Transaction on Antennas and Propogation, Vol. 53, No. 1, 569-571, 2005.

[8] Alkanhal, M. A. S. and A. F. Sheta, "A novel dual-band reconfigurable square-ring microstrip antenna,"Progress In Electromagnetics Research, PIER 70, 337-349, 2007.

[9] J. S. McLean, "A re-examination of the fundamental limits on the radiation Q of electrically small antennas," IEEE Trans. Antennas Propagat., vol. 44, pp. 672-676, May 1996.

[10] N. Behdad, and K. Sarabandi, "Bandwidth enhancement and further size reduction of a class of miniaturized slot antennas," IEEE Transaction on Antennas and Propogation, Vol. 52, No. 8, 2004.

[11] J. J. Lee, "Slotline impedance," IEEE Trans. Microwave Theory and Techniques, vol. 39, 666-672, Apr. 1991. 